\title{
Determinación de una técnica de referencia para la susceptibilidad antimicrobiana de bacterias anaeróbicas de importancia clínica
}

\author{
Sara I. Tapias', Betty de Galindo', Elizabeth Castañeda'
}

\begin{abstract}
Resumen
La selección de antibióticos para el tratamiento de infecciones por microorganismos anaeróbicos se hace en forma empírica en la mayoría de los casos; por tanto, el empleo de técnicas para determinar la susceptibilidad antimicrobiana es controvertido y sólo se realiza en centros de referencia. Con la aparición de cepas resistentes a los antimicrobianos de elección, es necesario determinar entre las técnicas estandarizadas una que pueda ser realizada en un laboratorio con cierta infraestructura para el trabajo en bacteriología clínica.
\end{abstract}

Se realizaron cuatro pruebas estandarizadas por el NCCLS: elución de disco en caldo, macrodilución, microdilución y dilución en agar, empleando cuatro cepas de referencia. Los datos obtenidos fueron analizados para determinar la reproducibilidad, la concordancia y la complejidad de las pruebas.

Empleando los criterios analizados, se obtuvo en orden descendente: en reproducibilidad, la dilución en agar, microdilución, macrodilución y elución; en concordancia, la dilución en agar, macrodilución, elución y microdilución; y en complejidad, dilución en agar, microdilución, macrodilución y elución.

Debido a la variedad de los resultados obtenidos no se pudo recomendar una sola técnica para la determinación de la susceptibilidad de microorganismos anaeróbicos de importancia clínica; en algunos casos y teniendo en cuenta el microorganismo y el antibiótico a probar, la realización de por lo menos dos de ellas permitiría esa determinación.

\section{Summary}

The selection of antibiotics ior the treatment of anaerobic infections is generally based on purely empirical criteria, with antimicrobial sensitivity tests being carried out only in reference centres. For this reason, it is important to investigate a technique appropriate for use in less specialized clinical bacteriology laboratories.

In this study, four antimicrobial sensitivity tests (elution in a broth disk, macrodilution, microdilution and dilution in agar) were carried out using four reference strains with known sensitivity patterns. The reproducibility, concordance, speed and ease of use of each test was compared.

1 Laboratorio de Microbiología, INS. 
The results obtained showed that the most reproducible test was dilution in agar, followed by microdilution, macrodilution and elution. In terms of concordance the best test was dilution in agar, followed by macrodilution, elution and microdilution. The most complex test was dilution in agar, followed by microdilution, macrodilution and elution.

As this study did not find any single test which, alone, proved better than any other in all the criteria analized, we recommend that a combination of at least two tests be used in the study of antimicrobial susceptibility of anaerobes.

Con el descubrimiento de la penicilina por Fleming en 1929, se inició el estudio y el análisis de la susceptibilidad antimicrobiana de los microorganismos facultativos de interés clínico; no así el de los microorganismos anaeróbicos debido principalmente a la dificultad para su aislamiento e identificación (1).

En los últimos años se ha incrementado el conocimiento sobre los microorganismos anaerobios, por el número de casos clínicos estudiados, y ha sido necesario el estudio de su comportamiento frente a los diferentes agentes antimicrobianos debido especialmente a que se han informado fallas en algunos tratamientos con las drogas consideradas de elección (2-4). Por tanto, se han desarrollado y estandarizado varios métodos y técnicas para medir la susceptibilidad a los antibióticos de los microorganismos anaeróbicos clínicamente significativos (5-7).

Con base en los métodos de susceptibilidad antimicrobiana ya estandarizados, nuestro propósito fue el de realizar cuatro de ellos para determinar su reproducibilidad y la complejidad de su realización con el fin de recomendar su ejecución a los laboratorios clínicos con un nivel intermedio de complejidad para el trabajo en bacteriología clínica.

\section{Materiales y métodos}

Cepas: se emplearon cuatro cepas control: Bacteroides fragilis ATCC 25285, Bacteroides thetaiotaomicron ATCC 29741, Clostridium perfringens ATCC 13124 y Eubacterium lentum ATCC 43055. Las cepas fueron mantenidas en medio de carne (8).

Antibióticos: los antibióticos se trabajaron en dos formas de presentación: disco y polvo. Se emplearon discos de penicilina G $10 \mathrm{UI}$, carbenicilina $100 \mu \mathrm{g}$, ticarcilina $75 \mu \mathrm{g}$, piperacilina $100 \mu \mathrm{g}$, mezlocilina $75 \mu \mathrm{g}$, ampicilina/sulbactam $10 \mu \mathrm{g}$, tetraciclina $30 \mu \mathrm{g}$, cefoxitin $30 \mu \mathrm{g}$, cefotetan $30 \mu \mathrm{g}$, cefoperazona $75 \mu \mathrm{g}$, cefotaxima $30 \mu \mathrm{g}$, moxalactam $30 \mu \mathrm{g}$ y cloranfenicol $30 \mu \mathrm{g}$ (todos de la casa Difco), metronidazol $80 \mu \mathrm{g}$ (preparados en el laboratorio), imipenen $10 \mu \mathrm{g}$ (BBL) y clindamicina $10 \mu \mathrm{g}$ (Oxoid). Los antibióticos en polvo fueron: penicilina $\mathrm{G}$, carbenicilina, ticarcilina, piperacilina, tetraciclina, cefoxitin, cefoperazona, cefotazime, moxalactan y cloranfenicol (todos de la marca Sigma), metronidazol (OFA-MK), ampicilina/ sulbactam (Pfizer), y clindamicina (Upjohn). Todos los antibióticos se conservaron a temperaturas de $2-8^{\circ} \mathrm{C}$.

\section{Pruebas de susceptibilidad}

1. Prueba de elución de disco: la prueba se realizó con pequeñas variaciones comparada con la técnica de elución descrita por el Comité Nacional para Estándares de Laboratorio Clínico (NCCLS) $(9,10)$. El inóculo se preparó con una turbidez igual al 0,5 de MacFarland (108 UFC/mL). Las variaciones fueron en el volumen del caldo, que fue de 5 $\mathrm{mL}$ para el tubo que contiene los discos y de $2,5 \mathrm{~mL}$ para los demás tubos y el inóculo fue de $0,025 \mathrm{~mL}$ para todos los tubos.

2. Prueba de macrodilución: se realizó según las recomendaciones del NCCLS (11).

3. Prueba de microdilución: se realizó según el NCCLS (11).

4. Dilución en agar: se realizó según el NCCLS (11).

\section{Evaluación de los resultados}

De cada una de las pruebas realizadas con las cuatro bacterias analizadas, se determinó el porcen- 
The results obtained showed that the most reproducible test was dilution in agar, followed by microdilution, macrodilution and elution. In terms of concordance the best test was dilution in agar, followed by macrodilution, elution and microdilution. The most complex test was dilution in agar, followed by microdilution, macrodilution and elution.

As this study did not find any single test which, alone, proved better than any other in all the criteria analized, we recommend that a combination of at least two tests be used in the study of antimicrobial susceptibility of anaerobes.

Con el descubrimiento de la penicilina por Fleming en 1929, se inició el estudio y el análisis de la susceptibilidad antimicrobiana de los microorganismos facultativos de interés clínico; no así el de los microorganismos anaeróbicos debido principalmente a la dificultad para su aislamiento e identificación (1).

En los últimos años se ha incrementado el conocimiento sobre los microorganismos anaerobios, por el número de casos clínicos estudiados, y ha sido necesario el estudio de su comportamiento frente a los diferentes agentes antimicrobianos debido especialmente a que se han informado fallas en algunos tratamientos con las drogas consideradas de elección (2-4). Por tanto, se han desarrollado y estandarizado varios métodos y técnicas para medir la susceptibilidad a los antibióticos de los microorganismos anaeróbicos clínicamente significativos (5-7).

Con base en los métodos de susceptibilidad antimicrobiana ya estandarizados, nuestro propósito fue el de realizar cuatro de ellos para determinar su reproducibilidad y la complejidad de su realización con el fin de recomendar su ejecución a los laboratorios clínicos con un nivel intermedio de complejidad para el trabajo en bacteriología clínica.

\section{Materiales y métodos}

Cepas: se emplearon cuatro cepas control: Bacteroides fragilis ATCC 25285, Bacteroides thetaiotaomicron ATCC 29741, Clostridium perfringens ATCC 13124 y Eubacterium lentum ATCC 43055. Las cepas fueron mantenidas en medio de carne (8).

Antibióticos: los antibióticos se trabajaron en dos formas de presentación: disco y polvo. Se emplearon discos de penicilina G $10 \mathrm{UI}$, carbenicilina $100 \mu \mathrm{g}$, ticarcilina $75 \mu \mathrm{g}$, piperacilina $100 \mu \mathrm{g}$, mezlocilina $75 \mu \mathrm{g}$, ampicilina/sulbactam $10 \mu \mathrm{g}$, tetraciclina $30 \mu \mathrm{g}$, cefoxitin $30 \mu \mathrm{g}$, cefotetan $30 \mu \mathrm{g}$, cefoperazona $75 \mu \mathrm{g}$, cefotaxima $30 \mu \mathrm{g}$, moxalactam $30 \mu \mathrm{g}$ y cloranfenicol $30 \mu \mathrm{g}$ (todos de la casa Difco), metronidazol $80 \mu \mathrm{g}$ (preparados en el laboratorio), imipenen $10 \mu \mathrm{g}$ (BBL) y clindamicina $10 \mu \mathrm{g}$ (Oxoid). Los antibióticos en polvo fueron: penicilina $\mathrm{G}$, carbenicilina, ticarcilina, piperacilina, tetraciclina, cefoxitin, cefoperazona, cefotazime, moxalactan y cloranfenicol (todos de la marca Sigma), metronidazol (OFA-MK), ampicilina/ sulbactam (Pfizer), y clindamicina (Upjohn). Todos los antibióticos se conservaron a temperaturas de $2-8^{\circ} \mathrm{C}$.

\section{Pruebas de susceptibilidad}

1. Prueba de elución de disco: la prueba se realizó con pequeñas variaciones comparada con la técnica de elución descrita por el Comité Nacional para Estándares de Laboratorio Clínico (NCCLS) $(9,10)$. El inóculo se preparó con una turbidez igual al 0,5 de MacFarland (108 UFC/mL). Las variaciones fueron en el volumen del caldo, que fue de 5 $\mathrm{mL}$ para el tubo que contiene los discos y de $2,5 \mathrm{~mL}$ para los demás tubos y el inóculo fue de $0,025 \mathrm{~mL}$ para todos los tubos.

2. Prueba de macrodilución: se realizó según las recomendaciones del NCCLS (11).

3. Prueba de microdilución: se realizó según el NCCLS (11).

4. Dilución en agar: se realizó según el
NCCLS (11).

\section{Evaluación de los resultados}

De cada una de las pruebas realizadas con las cuatro bacterias analizadas, se determinó el porcen- 
taje de reproducibilidad, concordancia y discrepancia. La reproducibilidad $(R)$ se calculó como el número de veces que la prueba dió los mismos resultados sobre el número de veces que la prueba fue realizada. La concordancia (C) se calculó como el número de veces que la prueba dió los resultados establecidos por la NCCLS sobre el número de veces que la prueba fue realizada. La discrepancia (D) se calculó como el número de veces que la prueba no dió los resultados establecidos por la NCCLS sobre el número de veces que la prueba fue realizada.

\section{Resultados}

Prueba de elución: la tabla 1 muestra que la técnica de elución fue más reproducible para $E$. lentum $(72 \%)$ y menos reproducible para $B$. thetaiotaomicron (47\%); mientras que se presentó mayor concordancia con C. perfringens $(94,6 \%)$ y menor con $B$. fragilis $(62,6 \%)$, esto último debido a que de los trece antibióticos probados, en tres no se logró obtener la CIM adecuada.

Prueba de macrodilución: la tabla 2 muestra que la técnica de macrodilución presentó mayor reproducibilidad y concordancia con $B$. thetaiotaomicron ( $74 \%$ y $100 \%$ respectivamente). Con C. perfringens a pesar de la baja reproducibilidad (54\%) se presentó una concordancia del $82 \%$.

Prueba de microdilución: la tabla 3 muestra que la técnica de microdilución fue más reproducible con $B$. fragilis ( $85 \%$ ) y menos reproducible con B. thetaiotaomicron (54\%); mientras que se presentó mayor concordancia con el $B$. thetaiotaomicron $(94,2 \%)$ y menos con $C$. perfringens $(65,9 \%)$ y $E$. lentum $(62,5 \%)$; esto fue debido, en el caso de $C$. perfringens, a que de los once antibióticos probados en uno no se logró obtener la CIM adecuada (según rangos de la NCCLS); mientras que con E. lentum de los seis antibióticos probados en dos no se logró obtener la CIM adecuada.

Prueba die dilución en agar: la tabla 4 muestra que la técnica de dilución en agar fue reproducible con B. fragilis ( $87 \%$ ) y menos reproducible con $C$. perfringens $(55 \%)$; mientras que la concordancia fue mayor con $B$. fragilis $(100 \%)$ y $E$. lentum (100\%), y la menor fue obtenida con $C$. perfingens $(87,1 \%)$.
Tabla 1. Porcentajes de reproducibilidad, concordancia y discrepancias de los datos obtenidos por la técnica de elución para las cuatro bacterias estudiadas.

\begin{tabular}{llccr}
\hline \multicolumn{1}{c}{ Bacteria } & $\mathbf{n}$ & $\begin{array}{c}\mathbf{R} \\
(\%)\end{array}$ & $\begin{array}{c}\mathbf{C} \\
(\%)\end{array}$ & $\begin{array}{c}\mathbf{D} \\
(\%)\end{array}$ \\
\hline B. fragilis & 50 & 66 & 62,6 & 37,3 \\
B. thetaiotaomicron & 46 & 47 & 73,9 & 26,0 \\
C. perfringens & 42 & 69 & 94,6 & 5,3 \\
E. lentum & 18 & 72 & 88,8 & 11,1 \\
\hline
\end{tabular}

Tabla 2. Porcentajes de reproducibilidad, concordancia y discrepancia de los datos obtenidos por la técnica de macrodilución para las cuatro bacterias estudiadas.

\begin{tabular}{lcccc}
\hline \multicolumn{1}{c}{ Bacteria } & $\mathbf{n}$ & $\begin{array}{c}\mathbb{R} \\
(\%)\end{array}$ & $\begin{array}{c}\mathbf{C} \\
(\%)\end{array}$ & $\begin{array}{c}\mathbf{D} \\
(\%)\end{array}$ \\
\hline B. fragilis & 42 & 66 & 87,8 & 12,1 \\
B. thetaiotaomicron & 39 & 74 & 100 & 0 \\
C. perfringens & 35 & 54 & 82,4 & 17,5 \\
E. lentum & 24 & 66 & 83,3 & 16,6 \\
\hline
\end{tabular}

Tabla 3. Porcentajes de reproducibilidad, concordancia y discrepancia de los datos obtenidos por la técnica de microdilución para las cuatro bacterias estudiadas.

\begin{tabular}{lcccc}
\hline \multicolumn{1}{c}{ Bacteria } & $n$ & $\begin{array}{c}R \\
(\%)\end{array}$ & $\begin{array}{c}\text { C } \\
(\%)\end{array}$ & $\begin{array}{c}\text { D } \\
(\%)\end{array}$ \\
\hline B. fragilis & 41 & 85 & 92,3 & 7,6 \\
B. thetaiotaomicron & 42 & 54 & 94,2 & 5,7 \\
C. perfringens & 44 & 56 & 65,9 & 34,0 \\
E. lentum & 24 & 70 & 62,5 & 37,5 \\
\hline
\end{tabular}

Tabla 4. Porcentajes de reproducibilidad, concordancia y discrepancia de los datos obtenidos por la técncia de dilución en agar para las cuatro bacterias estudiadas.

\begin{tabular}{lcccc}
\hline \multicolumn{1}{c}{ Bacteria } & $\mathbf{n}$ & $\begin{array}{c}\mathbf{R} \\
(\%)\end{array}$ & $\begin{array}{c}\mathbf{C} \\
(\%)\end{array}$ & $\begin{array}{c}\mathbf{D} \\
(\%)\end{array}$ \\
\hline B. fragilis & 39 & 87 & 100 & 0 \\
B. thetaiotaomicron & 39 & 71 & 92,2 & 7,6 \\
C. perfringens & 38 & 55 & 87,1 & 12,8 \\
E. lentum & 18 & 6 & 100 & 0 \\
\hline
\end{tabular}




\section{Discusión}

El estudio de la susceptibilidad antimicrobiana de los anaerobios puede realizarse por una serie de técnicas estandarizadas las cuales por su complejidad en su mayoría son realizadas por laboratorios de referencia(9-12). Unodelosobjetivos del trabajo fue el determinar de estas técnicas estandarizadas, una que pudiera ser utilizada en un laboratorio con cierta infraestructura para el trabajo de bacteriología clínica y así contribuir significativamente a la apropiada selección de agentes antimicrobianos para la terapia.

Las técnicas desarrolladas mostraron diversos grados de complejidad en su realización y diferentes niveles de reproducibilidad y concordancia, características que a su vez dependieron de la cepa y de los antibióticos empleados $(13,14)$.

Los problemas que se presentaron con $B$. fragilis para determinar la CIM de tres antibióticos (carbenicilina, ticarcilina y cefotaxima) con la técnica de elución habían sido informados previamente (14-17); por tanto, se recomienda utilizar otro de los métodos para esta bacteria (14). Otros antibióticos con los cuales se presentó dificultad para la determinación de la CIM para $B$. fragilis fueron la piperacilina y la penicilina G; se han informado recientemente problemas similares con la piperacilina por Aldridge y col. (15), a pesar de que en estudios anteriores realizados por Jorgensen se asegura que el método de elución es adecuado para probar la piperacilina (14). Con la penicilina $G$ no se han informado casos de dificultad en la determinación de la CIM.

Al realizar la técnica de elución con $B$. thetaiotaomicron se presentaron problemas similares a los informados en la literatura, en especial con la cefotaxima (15). Los problemas mencionados tuvieron como consecuencia la poca concordancia y la baja reproducibilidad de esta técnica para las especies de Bacteroides analizadas. Los resultados de la prueba de elución con $C$. perfringens y $E$. lentum presentaron buena concordancia, lo cual permite recomendarla como técnica adecuada para la determinación de la susceptibilidad a los antibióticos empleados. Se debe tener cuidado cuando se trabaja con un número grande de discos, ya que la desintegración de ellos puede confundirse con crecimiento bacteriano. Adicionalmente, es una técnica fácil de realizar por lo cual está al alcance de los laboratorios con una mediana complejidad en su estructura.

Los porcentajes de reproducibilidad obtenidos con la técnica de macrodilución fueron en general muy bajos; este hallazgo señala problemas técnicos los cuales se observaron cuando no ocurrió crecimiento de la cepa. Por el contrario, los porcentajes de concordancia fueron adecuados para todas las cepas lo que autoriza la recomendación de este procedimiento, aunque es algo dispendioso de realizar por la preparación de las diluciones y la necesidad de los antibióticos (materia prima) adecuados.

Con la técnica de microdilución se obtuvieron buenos resultados de reproducibilidad sólo para $B$. fragilis; los problemas técnicos fueron más aparentes con $B$. thetaiotaomicron, debido al no crecimiento del microorganismo. Sin embargo, la concordancia de los resultados con las especies de Bacteroides fue buena. La reproducibilidad y la concordancia obtenidas con C. perfringens y $E$. lentum fueron bajas. Los resultados con $E$. lentum pueden relacionarse con lo informado por Barry (13), que afirma que con este microorganismo se pueden obtener resultados diferentes, cuando se realizan pruebas de micro y macrodilución comparadas con la de dilución en agar, debido probablemente al uso de medios de cultivo diferentes. El método de la microdilución tiene la ventaja, cuando hay un número considerable de aislamientos, que las microplacas se pueden preparar con anterioridad y conservarse en congelación hasta su uso; también se pueden adquirir comercialmente.

Con la técnica de dilución en agar, el porcentaje de concordancia fue bueno para los cuatro microorganismos probados; la reproducibilidad obtenida por esta técnica fue variable, siendo buena para $B$. fragilis $B$. thetaiotaomicron, pero, baja para $C$. perfringens y $E$. lentum; debe tenerse en cuenta que de las cuatro técnicas ensayadas es la más dispendiosa de realizar y requiere un laboratorio con un nivel intermedio de complejidad, o uno de referencia. 
Agrupando las cuatro técnicas realizadas con base en los diferentes criterios analizados, se tendría en orden descendente: en reproducibilidad: la dilución en agar, microdilución, macrodilución y elución; en concordancia: la dilución en agar, macrodilución, elución y microdilución; y, en complejidad: dilución en agar, microdilución, macrodilución y elución.

Uno de los objetivos del trabajo fue el determinar, de los métodos estandarizados, uno de fácil realización, buena reproducibilidad y concordancia, el cual pudiera ser aplicado en laboratorios con cierta infraestructura para el trabajo de bacteriología clínica. Debido a la variación de los resultados obtenidos en nuestro estudio, la selección de un método único no fue posible; por tanto, se sugiere combinar dos de ellos (por ejemplo, el método de elución y macrodilución) dependiendo del microorganismo a investigar y los antibióticos a probar.

\section{Referencias}

1. Thonsberry C. Antimicrobial susceptibility testing of anaerobic bacteria: review, comments and opinions. Ann Otol Rhinol Laryngol 1991;154 suppl:7.

2. Finegold S. Gangrenous and/or perforated appendix: clinical outcome and in vitro susceptibility testing. Hosp Prac 1990;25:4 suppl:3.

3. Goldstein E, Citron D, Goldman R. National hospital survey of anaerobic, culture and susceptibility testing methods: results and recommendations for improvement. $\mathrm{J}$ Clin Microbiol 1992;30:1529.

4. Styrt B, Gorbach SL. Recent developments in the understanding of the pathogenesis and treatment of anaerobic infections. N Engl J Med 1989;321:298.

5. Barry AL, Fuchs PC, Allend SD, et al. Quality control limits for the standard anaerobic reference agar dilution susceptibility test procedure of the National Committee for Clinical Laboratory Standards. J Clin Microbiol 1989;27:192.
6. Finegold SM, Hannele R, Somer J, et al. Current perspectives on anaerobic infections: diagnostic approaches. Inf Dis Clin North America 1993;7:257.

7. Zabransky RJ, Abramson J, Barry AL, etal. Reference agar dilution procedure for antimicrobial susceptibility testing for anaerobic bacteria. NCCLS M11-A 1985;5:25.

8. Guzmán M, Galindo B. Bacteriología anaeróbica. En: Microbiología Médica. Manual de procedimientos. Serie de publicaciones científicas No. 14. Bogotá: Instituto Nacional de Salud, 1988:150.

9. Wexler HM, Finegoid SM. Antibacterial susceptibility tests: anaerobic bacteria. En: Balows A, Hausler WJ, Herrmann KL, Isenberg HD, Shadomy HJ, editors. Manual of clinical microbiology. Fifth edition. Washington, D.C: American Society for Microbiology, 1991:1133.

10. Zabransky RJ, Abrason J, Barry AL, Jones RN, Kurzynski, et al. Alternative methods for antimicrobial susceptibility testing of anaerobic bacteria. NCCLS M17P 1985;5:483.

11. Finegold SM, Albrige KE, Barry Al, et al. Methods for antimicrobial susceptibility testing of anaerobic bacteria. NCCLS 1993;3:1.

12. Schiro DD, Aldridge KE. Broth microdilution susceptibility testing of anaerobic bacteria. En: Isenberg HD, editor. Clinical microbiology procedures handbook. Washington, D.C.: American Society for Microbiology, 1992:1:5.6.

13. Barry A, Zabransky RJ. Eubacterium lentum ATCC 43055: a new reference strain for quality control of anaerobic susceptibility tests. J Clin Microbiol 1990;28:2375.

14. Jorgensen JH, Redding JS, Howell AW. Evaluation of broth disk methods for susceptibility testing of anaerobic bacteria with the newer B-lactam antibiotics. J Clin Microbiol 1986;23:545.

15. Aldridge K, Henderberg A, Schiro DD, Sanders CV. Discordant results between the broth disk elution and broth microdilution susceptibility tests with Bacteroides fragilis group isolates. J Clin Microbiol 1990;28:375.

16. Finegold S. Anaerobes: problems and controversies in bacteriology infections, and susceptibility testing. Rev Infect Dis 1990;12 suppl 2:223.

17. Wexler HM. Susceptibility testing of anaerobic bacteria: myth, magic, or method?. Clin Microbiol Rev 1991;4:470. 